\title{
CircRNA_09505 aggravates inflammation and joint damage in collagen-induced arthritis mice via miR-6089/AKT1/NF-KB axis
}

\author{
Jinghan Yang ${ }^{1}$, Min Cheng ${ }^{2}$, Bingjie $\mathrm{Gu}^{3}$, Jinghua Wang ${ }^{1}$, Shushan $\mathrm{Yan}^{4}$ and Donghua $\mathrm{Xu}^{1}$
}

\begin{abstract}
A number of circular RNAs (circRNAs) have been implicated in rheumatoid arthritis (RA) pathogenesis; however, little is known about their function and hidden molecular mechanism in immune and inflammation regulation. We investigated the role and the underlying mechanism of circRNA_09505 in RA in this study. Real-time PCR and fluorescence in situ hybridization (FISH) are adopted to estimate the quantitative expression and localization of circRNA_09505 in macrophages. The altering effect of circRNA_09505 on inflammation is investigated in vitro and in vivo by use of macrophage cell models and collagen-induced arthritis (CIA) mice. Luciferase reporter assay and RNAbinding protein immunoprecipitation (RIP) are used to confirm the circRNA_09505/miR-6089 ceRNA network predicted by bioinformatics analysis. Compared with controls, the expression of circRNA_09505 is upregulated in peripheral blood mononuclear cells (PBMCs) from patients with RA. The proliferation and cell cycle are significantly promoted when circRNA_09505 is upregulated in macrophages, whereas knockdown of circRNA_09505 inhibits macrophage proliferation and cell-cycle progression. Besides, circRNA_09505 can act as a miRNA sponge for miR-6089 in macrophages, and promote the production of TNF-a, IL-6, and IL-12 through ceRNA mechanism. Moreover, AKT1 is a direct target of miR-6089. CircRNA_09505 can promote AKT1 expression by acting as a miR-6089 sponge via IKBa/NFKB signaling pathway in macrophages. Most interestingly, knockdown of circRNA_09505 significantly alleviates arthritis and inflammation in vivo in CIA mice. These data support the hypothesis that circRNA_09505 can function as a miR6089 sponge and regulate inflammation via miR-6089/AKT1/NF-KB axis in CIA mice.
\end{abstract}

\section{Introduction}

Rheumatoid arthritis (RA) is a systemic autoimmune disease with unclear pathogenesis ${ }^{1}$. Inflammation and immunological disorders caused by macrophage, $\mathrm{B}$, and $\mathrm{T}$ lymphocytes contribute to $\mathrm{RA}^{2}$. Abnormal phenotype and function of those cells play crucial roles in regulating autoimmunity and inflammation. Both innate and

\footnotetext{
Correspondence: Shushan Yan (yanshushan@wfmc.edu.cn) or Donghua Xu (xudh@wfmc.edu.cn)

'Department of Rheumatology \& Central Laboratory of the First Affiliated Hospital, Weifang Medical University, Weifang 261053, China

${ }^{2}$ Department of Physiology, Clinical Medicine College, Weifang Medical University, Weifang 261053, China

Full list of author information is available at the end of the article These authors contributed equally: Jinghan Yang, Min Cheng, Bingjie Gu Edited by A. Stephanou
}

adaptive immune reactions are involved in the pathogenesis of RA. Macrophages are essential cells that promote systemic autoimmune disorders and chronic joint inflammation by linking innate and adaptive immunity in $\mathrm{RA}^{3,4}$. Increased level of macrophages in inflammatory lesions is a critical feature of RA, which infiltrates in synovial tissues and causes joint erosion. Macrophages have plasticity and pluripotency, which can differentiate into pro-inflammatory macrophages (called M1) and antiinflammatory macrophages (called M2) under diverse microenvironments ${ }^{5,6}$. Autoimmune disorders and excessive inflammatory responses perpetuate polarization of macrophages to a pro-inflammatory phenotype (M1), and thereby contribute to RA. As a result, depletion of 
macrophages from inflamed tissue is a promising strategy for RA treatment.

Mounting data have implicated that noncoding RNAs (ncRNAs) primarily including microRNA (miRNA), circular RNA (circRNA), and long ncRNA (lncRNA) play critical roles in mediating inflammation and immune regulation in autoimmune diseases ${ }^{7-9}$. Most interestingly, some ncRNAs encapsulated in extracellular vesicles can be found in peripheral circulation and confer modifying effects on joint lesions and tissue regeneration via cell-tocell communications in rheumatic diseases ${ }^{10,11}$. CircRNAs are stable ncRNAs with high evolutionary conservation. Emerging evidence has suggested that circRNAs are rich in binding sites of miRNAs and can sponge miRNAs through the competitive endogenous RNA (ceRNA) mechanism in complex biological processes, such as autoimmune imbalance, inflammatory response, and carcinogenesis ${ }^{12}$. Many specifically expressed circRNAs have been identified in RA, some of which regulate RA through ceRNA mechanism ${ }^{13,14}$. Nevertheless, the precise mechanism for circRNA/miRNA ceRNA network in RA has not been fully understood.

We have previously identified the specific profile of circRNA in RA patients by RNA sequencing. CircRNA_09505 is one of the most aberrantly expressed circRNAs in RA, which can also interact with miRNA through ceRNA mechanism predicted by bioinformatics analysis. The aim of this study is to explore the function and molecular mechanism of circRNA_09505 in vitro and in vivo by use of macrophage model and collagen-induced arthritis (CIA) mouse model.

\section{Results}

\section{Screening differentially expressed circRNAs in RA}

Differentially expressed circRNAs in peripheral blood mononuclear cells (PBMCs) from three patients and three controls were screened by RNA sequencing. As shown in Fig. 1a-c, there were 20 upregulated and 41 downregulated aberrantly expressed circRNAs in RA. Table 1 presents the top ten most significantly upregulated and downregulated circRNAs differentially expressed in RA, respectively. The top 20 dysregulated circRNAs were screened by RNA sequence and validated in PBMC samples from 36 patients and 30 controls by real-time polymerase chain reaction (PCR). Similar to the results of RNA sequencing, nine circRNAs were significantly upregulated, while eight circRNAs were significantly downregulated in RA PBMCs (Fig. 1d, e). Among them, circRNA_09505 was the most significantly upregulated one in PBMCs from RA patients. Most interestingly, the expression of circRNA_09505 was positively associated with ESR, CRP, and RF levels in serum from RA patients (Fig. 1f-h), suggesting significant relationship between circRNA_09505 expression and RA disease activity. Most importantly, the expression of circRNA_09505 was also found to be significantly increased in peripheral monocytes from RA patients compared with controls (Fig. 1i). GO and KEGG pathway enrichment analysis have suggested the differentially expressed circRNAs, including circRNA_09505 that might exert effects through the classic inflammation-related signaling pathways, such as NF- $\kappa B$ and TNF signaling pathways (Supplementary Fig. 1). Accordingly, we hypothesized that circRNA_09505 played a critical role in mononuclear macrophage-mediated inflammation in RA pathogenesis. In the following experiments, we further investigated the effect of circRNA_09505 and the underlying mechanism in regulating macrophages' inflammatory response in RA.

\section{CircRNA_09505 promoted macrophage proliferation and progression of cell cycle}

The expression of circRNA_09505 in macrophages was significantly increased when cells were transfected by circRNA_09505-overexpressed lentivirus plasmids (LVcircRNA) in contrast to the LV-NC group (Fig. 2a). Reversely, the expression of circRNA_09505 in macrophages transfected by sh-circRNA plasmids was reduced compared with sh-NC group (Fig. 2b). As demonstrated by fluorescence in situ hybridization (FISH), circRNA_09505 was mainly localized in the cytoplasm of macrophages (Fig. 2c). As a result, circRNA_09505 might influence macrophage proliferation and functions by interacting with other polypeptides, proteins, or nucleic acids that possess biological activity in the cytoplasm. First, we investigated the effect of circRNA_09505 on macrophage growth in vitro. As evidenced by EdU and CCK-8, the proliferation of macrophages was significantly increased when circRNA_09505 was overexpressed, but decreased when circRNA_09505 was knocked down in macrophages (Fig. $2 \mathrm{~d}-\mathrm{g}$ ). In addition, FACS showed that circRNA_09505 could also enhance the progression of the cell cycle of macrophages (Fig. 2h, i). Taken together, circRNA_09505 was capable of enhancing macrophage proliferation and cell-cycle progression in vitro.

\section{CircRNA_09505 regulated macrophage inflammatory response through circRNA_09505-miR-6089 ceRNA network}

Previously, we have demonstrated that miR-6089 was downregulated in PBMCs of RA patients and could regulate macrophage inflammatory response via TLR4 ${ }^{15}$. Here, the expression of miR-6089 was also found in microphage in RA patients (Fig. 3a). The negative association between circRNA_09505 and miR-6089 was observed in circRNA_09505-overexpressed macrophages (Fig. 3b). In addition, miR-6089 was predicted to be a targeted miRNA of circRNA_09505 by biological informatics analysis. MiR-6089 was capable of specifically 


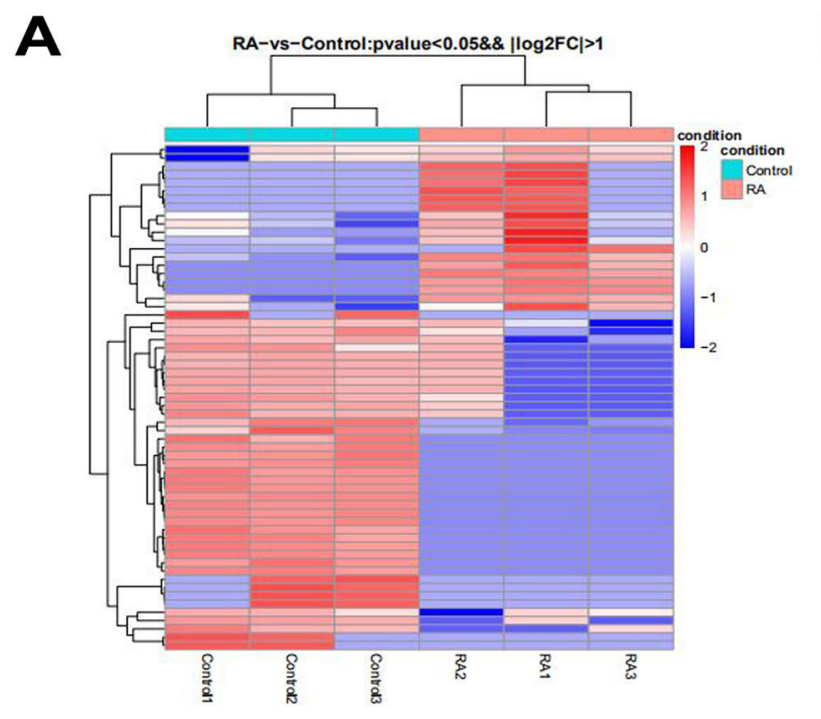

B
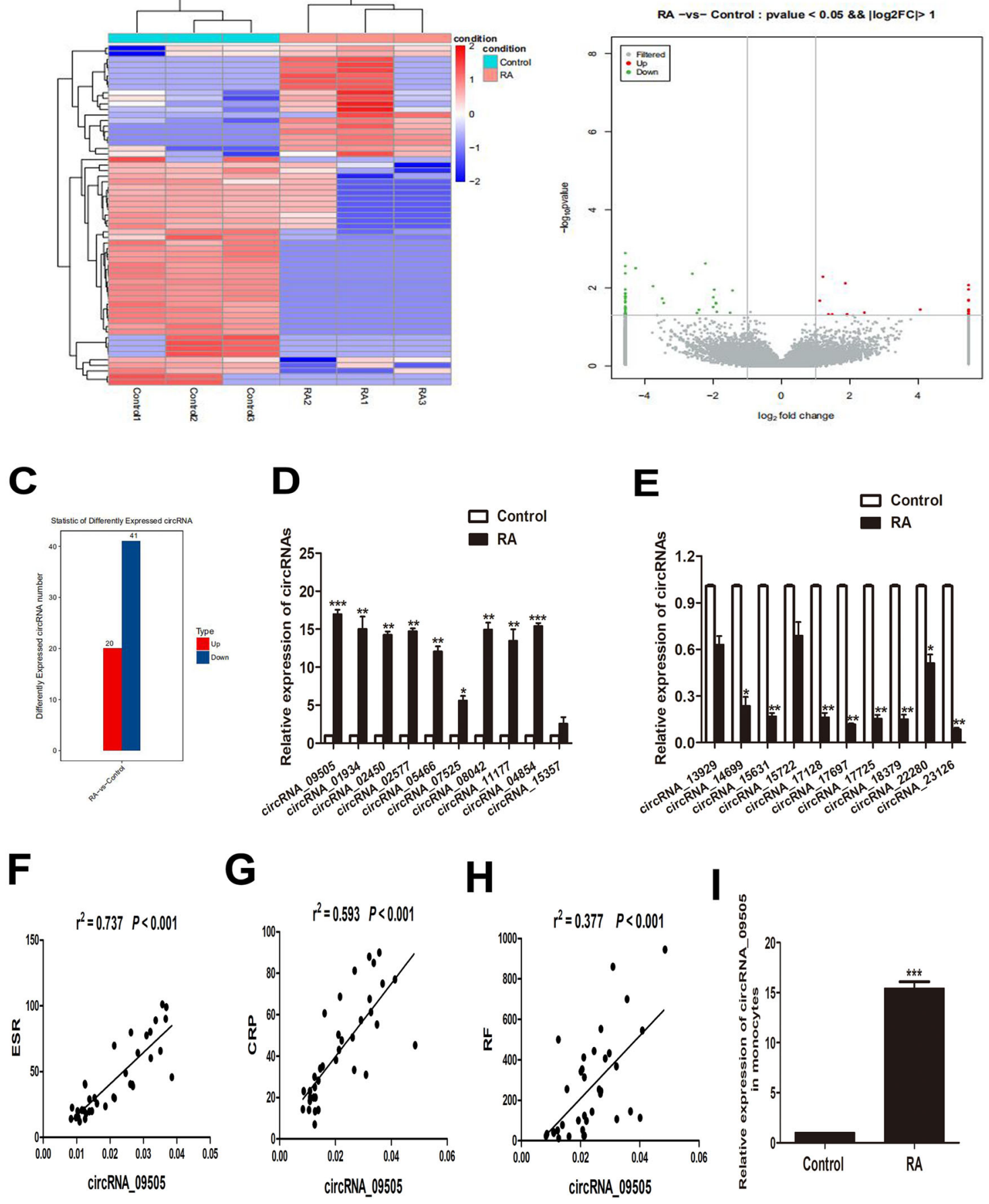

Fig. 1 CircRNA_09505 was aberrantly expressed and positively associated with ESR, CRP, and RF in RA. a Heat map. b Volcano scatter. c Column analyses showing differently expressed circRNAs in three RA patients compared with three healthy controls (up/down: 20/41, $P<0.05$, and | $\log 2 \mathrm{FC} \mid>1$ are considered as statistically significant). $\mathbf{d}$ Real-time PCR confirmed the expression of top 10 upexpressed circRNAs in PBMCs from 36 patients and 30 controls. e Real-time PCR confirmed the expression of top 10 downexpressed circRNAs in PBMCs from RA patients (RA/Control: 36/30). f CircRNA_09505 was positively associated with ESR in RA (RA: 36). g CircRNA_09505 was positively related to CRP in RA (RA: 36). h CircRNA_09505 was positively associated with RF in RA (RA: 36). i Increased circRNA_09505 expression in monocytes from RA patients compared with healthy controls (RA/Control: $21 / 18)\left({ }^{*} P<0.05,{ }^{* *} P<0.01,{ }^{* *} P<0.001\right.$; ESR erythrocyte sedimentation rate, CRP C-responsive protein, RF rheumatoid factor). 
Table 1 Top ten upregulated and downregulated aberrantly expressed circRNAs in RA PBMCs.

\begin{tabular}{|c|c|c|c|}
\hline CircRNAs & Fold & Change & $P$ \\
\hline circRNA_09505 & 18.002 & Up & 0.011 \\
\hline circRNA_01934 & 17.442 & Up & 0.020 \\
\hline circRNA_02450 & 17.316 & Up & 0.048 \\
\hline circRNA_02577 & 17.222 & Up & 0.036 \\
\hline circRNA_05466 & 17.215 & Up & 0.038 \\
\hline circRNA_07525 & 17.115 & Up & 0.036 \\
\hline circRNA_08042 & 16.490 & Up & 0.040 \\
\hline circRNA_11177 & 16.455 & Up & 0.008 \\
\hline circRNA_04854 & 16.322 & Up & 0.021 \\
\hline circRNA_15357 & 16.113 & Up & 0.040 \\
\hline circRNA_13929 & 17.991 & Down & 0.019 \\
\hline circRNA_14699 & 17.402 & Down & 0.032 \\
\hline circRNA_15631 & 17.389 & Down & 0.046 \\
\hline circRNA_15722 & 17.168 & Down & 0.046 \\
\hline circRNA_17128 & 17.117 & Down & 0.034 \\
\hline circRNA_17697 & 16.111 & Down & 0.017 \\
\hline circRNA_17725 & 15.088 & Down & 0.014 \\
\hline circRNA_18379 & 15.069 & Down & 0.014 \\
\hline circRNA_22280 & 14.621 & Down & 0.040 \\
\hline circRNA_23126 & 14.002 & Down & 0.016 \\
\hline
\end{tabular}

recognizing 13 bases of circRNA_0950 sequence (Fig. 3c). Figure 3d shows the potential circRNA_09505-miR6089-mRNA network, which was scanned in databases of Miranda and TargetScan. Given these findings, we hypothesized that circRNA_09505 could regulate macrophages by sponging miR-6089 through ceRNA mechanism. Moreover, the luciferase reporter assay had supported this hypothesis (Fig. 3e). In addition, RIP assay showed that both circRNA_09505 and miR-6089 were elevated in the immunoprecipitates of the anti-Ago2 group (Fig. 3f, g). Both circRNA_09505 and miR-6089 inhibitors were reduced in miR-6089 inhibitor-treated group compared with the IgG group (Fig. 3f, g). Accordingly, circRNA_09505 could function as a miR-6089 sponge in macrophages. In the following experiments, we investigated the influence of circRNA_09505/miR-6089 on macrophage-mediated inflammation in RA pathogenesis. CircRNA_09505 promoted the expression of TNF- $\alpha$, IL-6, and IL-12 mRNAs in macrophages (Fig. $3 \mathrm{~h}-\mathrm{j}$ ). The protein level of these cytokines in the cultural supernatant of macrophages was also increased when circRNA_09505 was overexpressed in cells (Fig. 3k-m). However, the rescue tests using miRNA mimics showed that miR-6089 mimics could suppress the effect of circRNA_09505 on macrophage inflammation (Fig. 3k-m). Taken together, circRNA_09505 regulated macrophage inflammatory response via miR-6089 sponge in vitro through ceRNA mechanism.

\section{CircRNA_09505-miR-6089 ceRNA target-regulated AKT1 depending on IKBa/NF-KB signaling pathway in macrophages}

To further investigate the targets of circRNA_09505-miR-6089 ceRNA network, we performed bioinformatics analysis in TargetScan database. Figure 4a shows that AKT1 was a potential targeted mRNA for miR-6089 because it had complementary bases in the $3^{\prime}$-untranslated region (UTR) recognized by miR6089. Negative association between AKT1 and miR-6089 was found in macrophages (Fig. 4b, c). Besides, the luciferase reporter assay supported that miR-6089 could downregulate the expression of AKT1, whereas miR-6089 inhibitors could restrain its effect (Fig. 4d). Taken together, AKT1 was the targeted gene of miR-6089. Moreover, circRNA_09505 could promote the expression of AKT1, $\mathrm{p}-\mathrm{I} \kappa \mathrm{B} \alpha$, and p-NF- $\mathrm{B}$ in macrophages (Fig. 4e). However, miR-6089 mimics could partially rescue this effect via circRNA_09505/miR-6089 sponge (Fig. 4e). These findings demonstrated that circRNA_09505 could act as a ceRNA by acting as a miR-6089 sponge and regulate inflammatory response through $\mathrm{AKT} 1 / \mathrm{NF}-\mathrm{k} B$ signaling pathway in macrophages.

\section{Blocking circRNA_09505 in macrophages alleviated inflammation and joint damage in CIA mice}

CIA mice model was constructed following the flowchart in Fig. 5a. Blocking circRNA_09505 in macrophages significantly reduced the mean arthritis score and prolonged the time of arthritis that first appeared compared with the control mice (Fig. 5b). Less severe joint injury, reduced subchondral bone erosions, and lymphocyte infiltration in tissues were observed in sh-circRNAtransfected macrophage-treated mice (Fig. 5c, d). Knockdown of circRNA_09505 in macrophages protected CIA mice from severe bone destruction as seen in histomorphometric assays (Fig. 5e, f). Synovitis and cartilage damage of CIA mice were obviously alleviated when they were treated with circRNA_09505-blocked macrophages (Fig. 5g). circRNA_09505 was decreased in PBMCs and synovium of CIA mice administered with sh-circRNA plasmid-transfected macrophages (Fig. 6a, b). F4/80 macrophages were less infiltrated in mice synovial tissues when they were treated with circRNA_09505 knocked-out macrophages (Supplementary Fig. 2). The expression of IL- 6 and TNF- $\alpha$ mRNA in mice PBMCs was significantly decreased after blocking circRNA_09505 in macrophages (Fig. 6c, d). Cytokines of IL-6 and TNF- $\alpha$ in mouse serum 

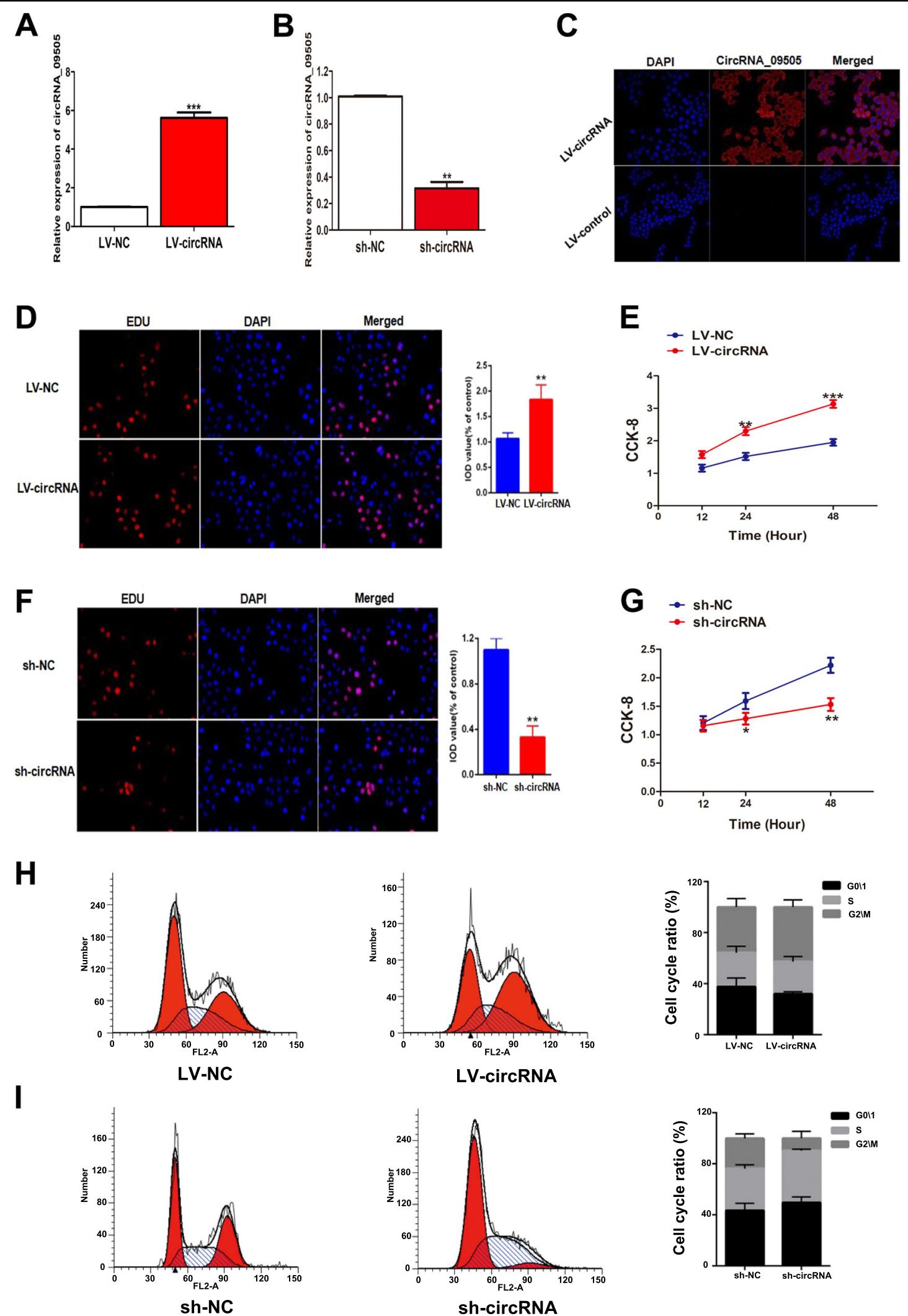

Fig. 2 (See legend on next page.) 
(see figure on previous page)

Fig. 2 CircRNA_09505 enhanced macrophage proliferation and cell-cycle progression. a Real-time PCR showed that circRNA_09505 was increased in LV-circRNA-transfected macrophages. b Real-time PCR showed that circRNA_09505 was decreased in sh-circRNA-transfected macrophages compared with controls. c Representative FISH picture showed that circRNA_09505 was primarily localized in the cytoplasm of macrophages. $\mathbf{d}$ Representative scanning picture of EdU showing increased proliferation of LV-circRNA_09505-transfected macrophages compared with control group. e CCK-8 showed that the proliferation of LV-CircRNA_09505-transfected macrophages was increased. $\mathbf{f}$ Representative scanning picture of EdU showing decreased proliferation of sh-circRNA_09505-transfected macrophages compared with control group. g CCK-8 showed decreased proliferation of sh-circRNA_09505-transfected macrophages. $\mathbf{h}$ The cell cycle of LV-circRNA_09505-transfected macrophages was estimated by flow cytometry. i The cell cycle of sh-circRNA_09505-transfected macrophages was estimated by flow cytometry. We calculated cell percentages in G0/G1, S, and G2/M phases, respectively. All data represented three independent tests. ${ }^{*} P<0.05$, ${ }^{* *} P<0.01,{ }^{* * *} P<0.001$.

were also obviously reduced in mice that were treated with circRNA_09505 knocked-down macrophages (Fig. 6e, f). TNF- $\alpha$ was decreased in mouse synovial tissue macrophages that were treated with circRNA_09505 knocked-down macrophages, while IL-4 in macrophages was increased (Supplementary Fig. 3). In addition, the expression of p-AKT1 and p-NF-kB in mouse synovial tissues was determined by immunohistochemistry. The phosphorylation and activation of AKT1 and NF- $\mathrm{kB}$ in joint synovial tissue macrophages were significantly inhibited in CIA mice treated with circRNA_09505blocking macrophages (Fig. 6g, h). As a result, it could be concluded that circRNA_09505 could influence the inflammation of CIA mice in vivo. Blocking circRNA_09505 in macrophages could alleviate inflammation and joint damage in CIA mice.

\section{Discussion}

A growing body of data have implicated that ncRNAs are key regulators for coding genes at transcriptional or post-transcriptional levels in a variety of autoimmune diseases including $\mathrm{RA}^{7,12,16}$. CircRNAs have been initially identified as ncRNAs and gradually highlighted in multiple physiological and pathophysiological processes ${ }^{12}$. A large body of data has suggested the pivotal role of circRNAs in regulating autoimmunity and inflammation. There is sufficient evidence that a number of circRNAs are aberrantly expressed in RA, which may participate in RA pathogenesis ${ }^{17,18}$. Our study shows the profile of circRNAs in PBMC samples from RA patients by RNA sequencing. Besides, we have first demonstrated that circRNA_09505 is the most significantly increased circRNA in RA PBMCs, which can function as miR-6089 sponge and regulate macrophage inflammation by targeted regulation of AKT1 in vitro. Moreover, blocking circRNA_09505 in macrophages alleviates inflammation and joint damage in CIA mice in vivo. As a result, circRNA_09505-miR-6089 ceRNA network would serve as novel targets for RA diagnosis and treatment.

CeRNA is well known as a mechanism about the interaction between different RNAs. It has been well established that miRNA can result in gene silencing by binding to mRNA, whereas ceRNA can regulate mRNA and affect gene silencing caused by miRNA by competing with it ${ }^{19}$. CeRNA cross talk has been documented to contribute to disease pathogenesis, such as cancer, inflammation, and autoimmunity ${ }^{20,21}$. Mounting studies have shown the evidence that a variety of RNAs, including circRNAs, lncRNAs, mRNAs, and pseudogenes, can function as miRNA sponge via ceRNA mechanism in various biologic processes. In particular, some available studies have reported the sequencing and microarray technologies to investigate the dysregulated transcripts in certain cells or whole blood of RA patients, some of which are demonstrated to be functional ceRNAs in $\mathrm{RA}^{22,23}$. We have previously found that lncRNA HIX003209 is dysregulated in RA, which functions as ceRNA for miR-6089 and regulates inflammation by targeting TLR4 in macrophages $^{24}$. Accordingly, ncRNAs play a crucial role in RA pathogenesis by ceRNA cross talk.

CircRNAs belong to a family of endogenous ncRNAs without $5^{\prime}$ caps or $3^{\prime}$ tails, which have been implicated in inflammation and immune regulation. Apart from interacting with molecules with biological activity directly, a number of circRNAs have also been confirmed to function as ceRNA in cancer, inflammation-related, or autoimmune diseases ${ }^{25-27}$. Although several published studies have identified the specific profile of circRNAs in peripheral blood cells of RA patients, whether those circRNAs can function as ceRNA for the dysregulated miRNAs in RA is not clear yet. We, for the first time, demonstrate that dysfunction of circRNA_09505 contributes to RA by regulating macrophage proliferation, cell cycle, and inflammatory response. In addition, it is involved in macrophage inflammation through circRNA_09505-miR-6089 ceRNA network. CircRNA_09505 can promote AKT1 expression and NF-kB activation in macrophages in vitro, although miR-6089 mimics can partially rescue this effect via circRNA_09505/miR-6089 sponge. Moreover, knockdown of circRNA_09505 in macrophages alleviates inflammation and joint damage in CIA mice in vivo. As a result, circRNA_09505 may be a potential target for RA treatment in combination with macrophage therapy. Nonetheless, 


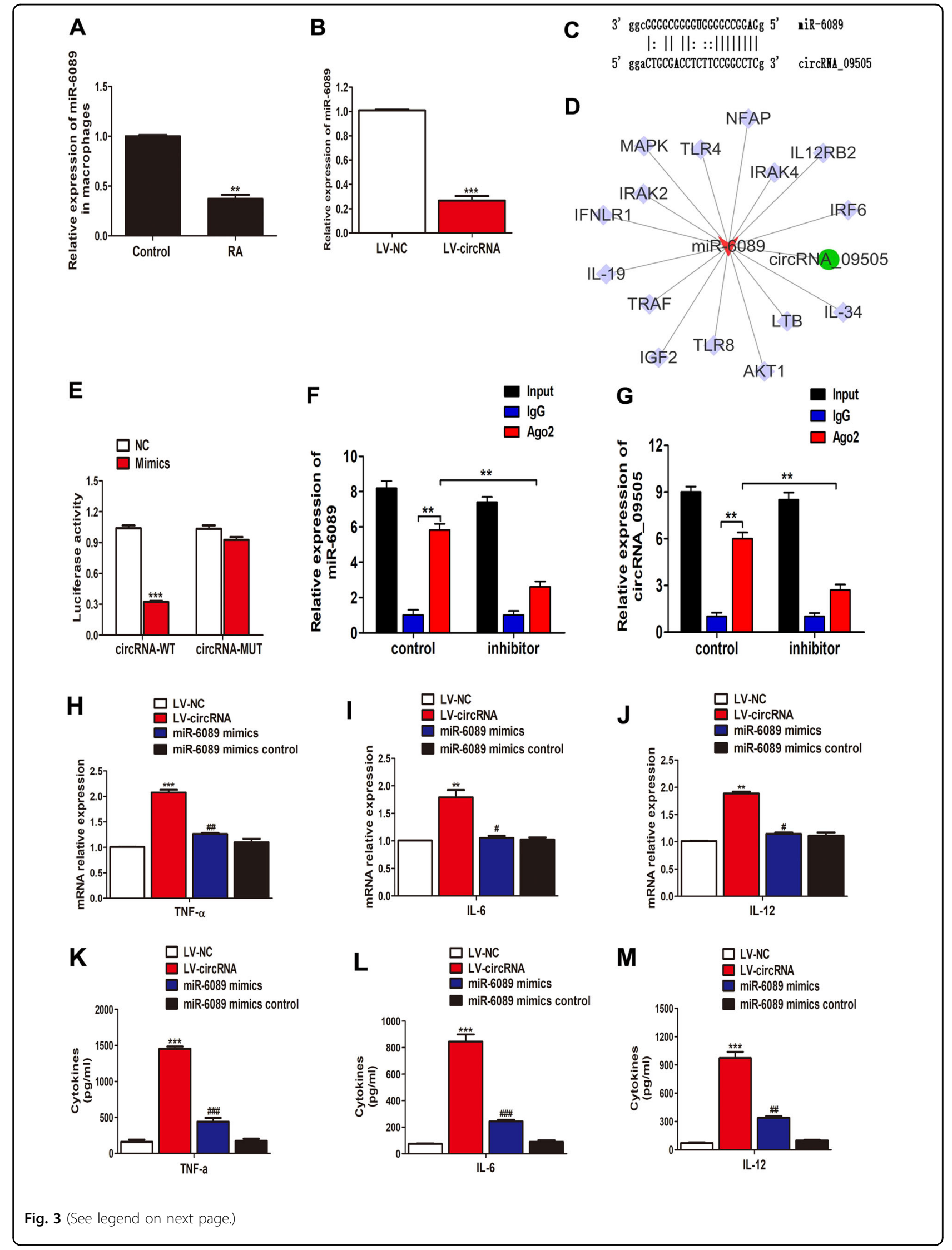


(see figure on previous page)

Fig. 3 CircRNA_09505-miR-6089-mRNA network in macrophages. a Decreased miR-6089 expression in macrophages from RA patients compared with healthy controls (RA/Control: 21/18, ${ }^{* *} P<0.01$ ). b Reduced expression of miR-6089 in LV-circRNA_09505-transfected macrophages as evidenced by real-time PCR ${ }^{* * *} P<0.001$, data of three independent experiments). c Complementary bases of circRNA_09505 and miR-6089. d Targeted mRNAs of circRNA_09505-miR-6089 axis scanned in databases of Miranda and TargetScan. e Luciferase reporter assay demonstrated that miR-6089 was a targeted miRNA of circRNA_09505 (WT wild type, MUT mutant type, compared with normal control (NC) group, ${ }^{* * *} P<0.001$; representative data of three repeated tests in vitro). $\mathbf{f}$ RIP assay was performed using input, IgG, or anti-Ago2 antibodies. Relative expression of miR6089 assayed by real-time PCR. $\mathbf{g}$ RIP assay and relative expression of circRNA_09505 determined by real-time PCR. Data of three independent assays. ${ }^{*} P<0.01$. h Real-time PCR showed TNF-a mRNA expression in macrophages of four groups (cells treated by LV-NC plasmids, LV-circRNA plasmids, miR-6089 mimics plus LV-circRNA plasmids, and miR-6089 mimic controls plus LV-NC plasmids, respectively). i Real-time PCR showed IL-6 mRNA expression in macrophages. $\mathbf{j}$ Real-time PCR showed IL-12 mRNA expression in macrophages. $\mathbf{k}$ ELISA demonstrated TNF-a production in the supernatant of macrophages. I ELISA demonstrated IL-6 production in the supernatant of macrophages. $\mathbf{m}$ ELISA demonstrated IL-12 production in the supernatant of macrophages. $\mathbf{k}-\mathbf{m}$ Compared with the LV-NC group, ${ }^{* * *} P<0.001$; compared with LV-circRNA group, ${ }^{\#} P<0.05,{ }^{\# \#} P<0.01,{ }^{\# \# \#} P<$ 0.001; data of three independent tests.
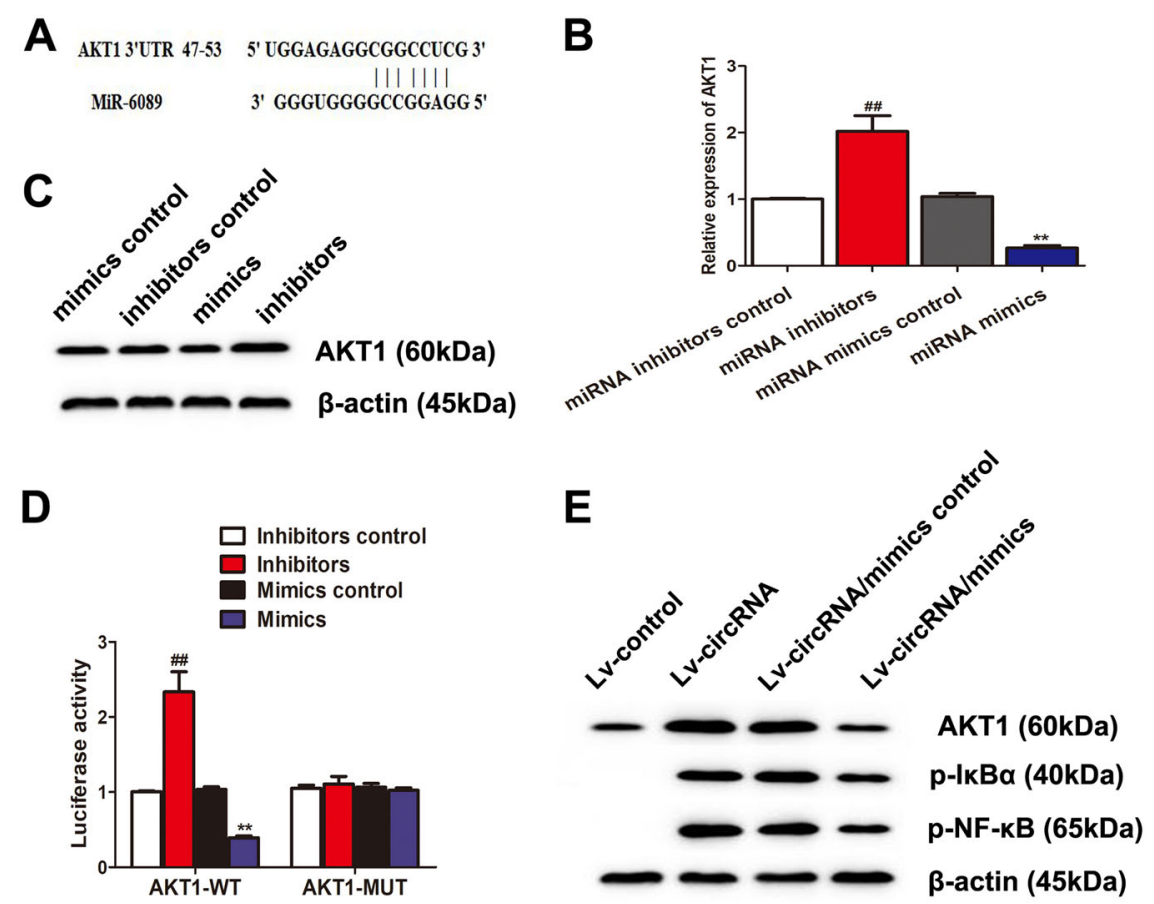

Fig. 4 CircRNA_09505 regulated AKT1 via sponging miR-6089. a Complementary sequences of the 3'UTR of AKT1 recognized by miR-6089. b MiR-6089 inhibited the expression of AKT1 mRNA in macrophages as evidenced by real-time PCR. c Representative western blot estimating AKT1 protein. $\mathbf{d}$ Luciferase reporter assay demonstrated that AKT1 was a target of miR-6089, but the rescue test by use of miR-6089 inhibitors reversed its effect on AKT1. e The western blot analysis showed that circRNA_09505 increased the expression of AKT1, p-IKBa, and p-NF-KB in macrophages, while miR-6089 mimics partially restrained its effect through ceRNA mechanism. In contrast to miRNA mimic control group, ${ }^{*} P<0.01$; compared with miRNA inhibitor control group, ${ }^{\#} P<0.01$. Experiments were repeated at least three times.

more investigations are warranted to apply it to RA treatment in the future.

Macrophages are key cells that participate in inflammatory and immune reactions, which play a vital role in RA. Elevated level of macrophages can infiltrate in the inflamed synovium tissue and cause inflammatory lesions and joint damage in $\mathrm{RA}^{3}$. Some circRNAs are reported to be specifically expressed in macrophages and affect their differentiation, infiltration, activation, and function in particular tissues ${ }^{28-30}$. There are different expression patterns of circRNAs in diverse polarized macrophages primarily including pro-inflammatory $\mathrm{M} 1$ and antiinflammatory M2 macrophages ${ }^{28}$. However, the mechanism by which circRNAs regulate macrophage polarization and function particularly in RA remains obscure. The present study shows the specific expression profile of circRNAs in PBMCs from RA patients, while the expression of circRNAs in polarized macrophages and 


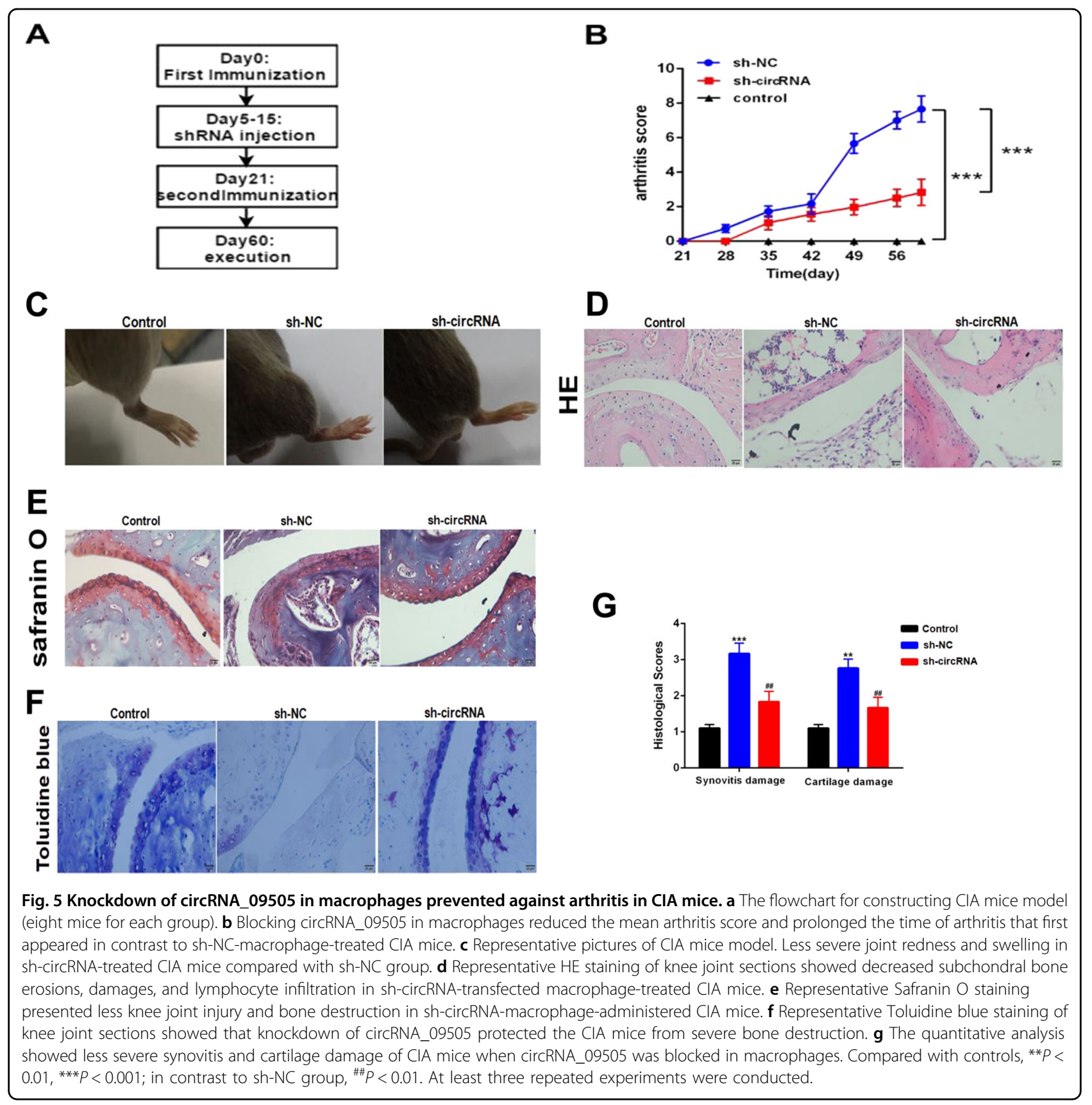

their molecular mechanism is not elucidated. Investigating the mechanism that drives macrophage polarization toward M1 or M2 phenotypes is worthy to be done, because depletion of macrophages with pro-inflammatory phenotype like circRNA_09505 from the inflamed tissue is a promising therapeutic strategy for RA. In this study, we have demonstrated the modifying effect of circRNA_09505 on macrophages in vitro. CircRNA_09505 aggravates macrophage inflammation by promoting the generation of inflammatory cytokines TNF- $\alpha$, IL-6, IL-8, IL-12, and IL-1 $\beta$, which are most commonly dysregulated cytokines in RA. In addition, TNF- $\alpha$ in synovial macrophages is significantly decreased when CIA mice are treated with circRNA_09505-blocking macrophages through tail-vein injection. However, whether circRNA_09505 regulates macrophage differentiation and polarization in vivo is unclear. Accordingly, more studies, particularly in vivo research, are encouraged to elucidate the role of circRNA_09505 in macrophage differentiation and function in the future.

To summarize, circRNA_09505 regulated macrophage inflammation by functioning as a ceRNA for miR-6089 
A

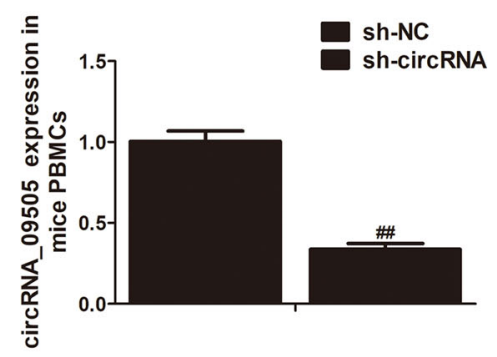

C
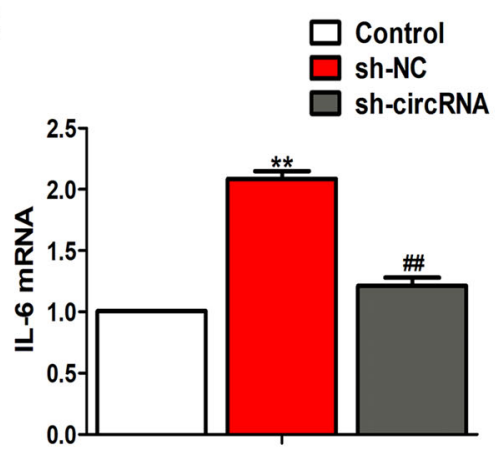

E
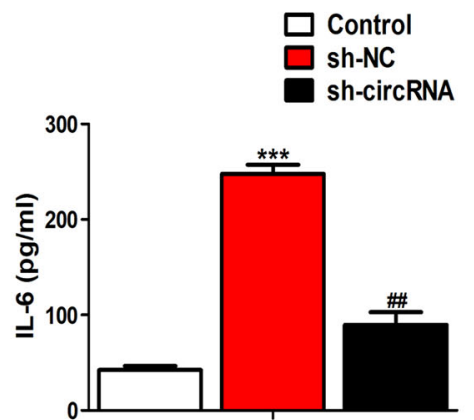

G

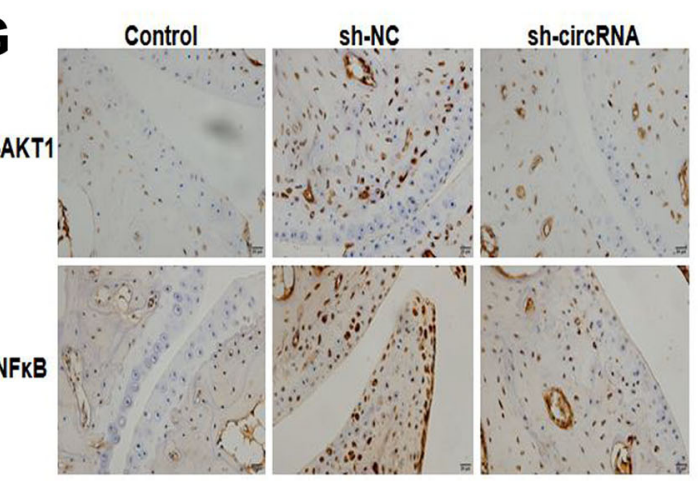

B

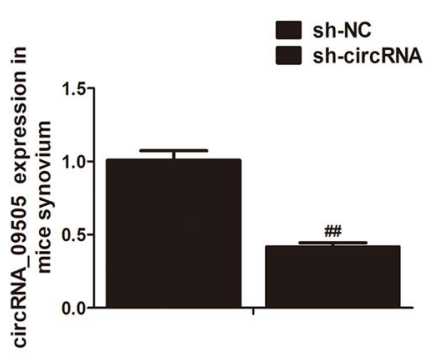

D
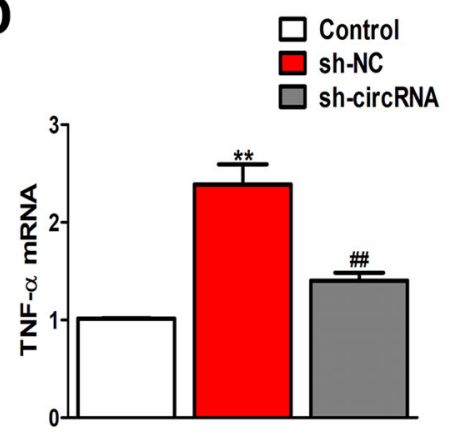

F

$\square$ Control

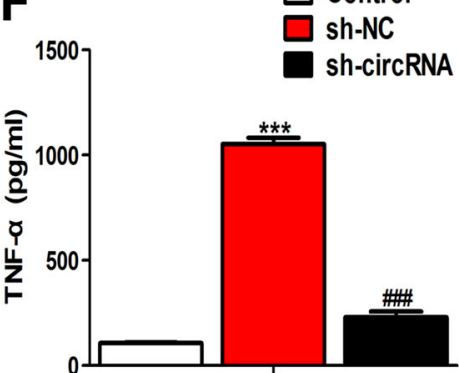

H

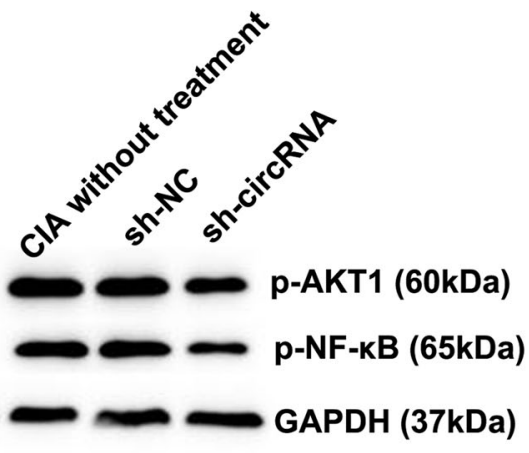

Fig. 6 Knockdown of circRNA_09505 in macrophages alleviated inflammation via AKT1/NF-KB signaling pathway in CIA mice. a Decreased expression of circRNA_09505 in PBMCs of CIA mice treated with sh-circRNA plasmid- transfected Raw264.7 macrophages compared with CIA mice treated with sh-NC plasmid-transfected macrophages. b Reduced expression of circRNA_09505 in synovium of CIA mice determined by real-time PCR. c Knockdown of circRNA_09505 in macrophage-inhibited IL-6 mRNA expression in PBMCs from CIA mice. d Knockdown of circRNA_09505 in macrophage-inhibited TNF-a mRNA expression in PBMCs from CIA mice. e Knockdown of circRNA_09505 in macrophages decreased IL-6 in mouse serum. $\mathbf{f}$ Knockdown of circRNA_09505 in macrophages decreased TNF-a protein in mouse serum. $\mathbf{g}$ Representative immunohistochemistry sections showing decreased expression of p-AKT1 and p-NF-KB in mouse knee joint synovial tissues. $\mathbf{h}$ Representative western blot pictures showing reduced expression of p-AKT1 and p-NF-KB in mouse knee joint synovial tissue macrophages of CIA mice. Compared with normal controls (NC), ${ }^{* *} P<0.01$, ${ }^{* * *} P<0.001$; in contrast to sh-NC group, ${ }^{\# \#} P<0.01 ;{ }^{\# \# \#} P<0.001$. At least three repeated experiments were conducted. 
through AKT1/NF-kB signaling pathway. Blocking circRNA_09505 in macrophages can reduce inflammation and joint damage in CIA mice. These findings show strong evidence supporting the use of circRNA_09505 as a target for RA immune therapy. Nevertheless, more mechanisms and function-associated experiments are warranted to explore it as a useful treatment strategy for RA in the future.

\section{Materials and methods}

\section{Patients, controls, and animals}

There are 36 initially diagnosed and untreated RA patients and 30 age- and gender-adjusted healthy controls from the First Affiliated Hospital of Weifang Medical University. The characteristics of the participants are shown in Table 2. They have read and signed the written informed consent before peripheral blood sample collection. The present study is approved and supervised by the Institutional Ethics Committee of the First Affiliated Hospital, Weifang Medical University. We extract PBMCs from patients and controls according to the protocol of human lymphocytes isolation solution (TBD, Tianjin, China) by gradient centrifugation at $1800 \mathrm{~g}$ for $30 \mathrm{~min}$. PBMCs and serum samples are also stored at $-20^{\circ} \mathrm{C}$ for subsequent use. In addition, peripheral blood monocytes are isolated from $21 \mathrm{RA}$ patients and 18 age- and genderadjusted healthy controls by CD14 microbeads (Miltenyi Biotec, San Diego, USA) according to the protocol. C57BL/6 (8 weeks old) male mice are purchased from Pengyue Experimental Animal Breeding Co., Ltd. (Jinan, Shandong Province, China), which are used to construct CIA mice model. Mice were randomly divided in this study. All animal experiments are carried out based on the Guideline of Institutional Animal Care and Use Committee. About $4 \mathrm{mg} / \mathrm{ml}$ bovine type II collagen (Chondrex, Washington, USA) is dissolved in $50 \mathrm{mM}$ acetic acid and emulsified with Freund's complete adjuvant (SigmaAldrich, USA) of equal volume by repeated suction. Eight mice in each group are immunized by use of $100 \mu \mathrm{l}(4 \mathrm{mg} /$

Table 2 Summary characteristics of RA patients and controls.

\begin{tabular}{lll}
\hline & Control (30) & RA (36) \\
\hline Age (yrs) & $23-60$ & $25-63$ \\
Sex (M/F) & $5 / 20$ & $6 / 22$ \\
Tobacco smoking (yr) & $15.0 \pm 2.7$ & $18.1 \pm 3.2$ \\
Alcohol consumption (yr) & $9.2 \pm 1.5$ & $10.3 \pm 2.0$ \\
ESR (mm/h) & $12.0 \pm 1.8$ & $48.1 \pm 2.2$ \\
CRP (mg/L) & $7.0 \pm 1.6$ & $40.9 \pm 3.2$ \\
RF (IU/ml) & $10.9 \pm 2.0$ & $71.5 \pm 7.6$ \\
\hline
\end{tabular}

$\mathrm{ml}$ ) of emulsion solution through intradermal injection at the base of the tail (day 0). Booster injection using $100 \mu \mathrm{l}$ of emulsion solution is conducted by intradermal injection at another site of the tail base on day 21 . In the treatment study, mice with the score reaching six are randomly separated into sh-circRNA-treated Raw264.7 macrophage group and sh-NC-treated macrophage group. Mice were intravenously injected with plasmidtransfected macrophages through the tail vein on days 5 and 15 , respectively. The longest time for mice arthritis estimation is 60 days from the first immunization. Mouse PBMCs are isolated from eyeball blood using mouse lymphocytes isolation solution (TBD, Tianjin, China). Synovial macrophages are isolated from mouse joints according to the protocol of mouse tissue lymphocytes isolation solution (TBD, Tianjin, China) and anti-mouse F4/80 monoclonal antibody (Miltenyi Biotec, San Diego, USA). All samples and isolated cells are stored at $-20^{\circ} \mathrm{C}$ for determinations. Animal studies are approved by the Animal Ethics Committee of Weifang Medical University.

\section{RNA sequence}

PBMC samples from three RA and three healthy controls were analyzed by high-throughput sequencing by Oebiotech Company (Shanghai, China) to screen the aberrantly and specially expressed circRNAs in RA. The co-expression of mRNAs in PBMCs from RA patients was also estimated. Prediction of potential target genes and signaling pathway of circRNAs were also adjusted by bioinformatics analysis in databases of Miranda and TargetScan.

\section{Cell culture and transfection}

PMA (100 nM) was used to stimulate THP- 1 for $48 \mathrm{~h}$ to make them differentiate into macrophage-like cells. THP1 and Raw264.7 macrophages were cultured in DMEM (Sigma-Aldrich, USA) with $10 \%$ fetal bovine serum (Gibco, USA) at $37^{\circ} \mathrm{C}, 5 \% \mathrm{CO}_{2}$. MiRNA inhibitors, mimics, and controls are purchased from Ruibo Biosciences (Guangzhou, China). PcDNA3.1 lentivirus products LV-circRNA, sh-circRNA, and the corresponding negative controls are constructed in 293T cells using Lipofectamine 2000 (Invitrogen, NY, USA). Polybrene reagent is used to transfect macrophages. Stably transfected cells are finally verified by quantitative realtime PCR.

\section{Quantitative real-time PCR}

The expression profile of circRNA in PBMCs of RA patients is screened by RNA sequencing in Oebiotech Company (Shanghai, China). Quantitative real-time PCR is applied to confirm the differentially expressed circRNAs and miR-6089 in RA. TRIzol (Invitrogen, CA, USA) is used for RNA isolation. About $0.5 \mu \mathrm{g}$ of RNAs are used as 
a model for cDNA synthesis according to the protocol of Takara RT kit (Tianjin, China). About 5 ng of cDNAs are used as templates for PCR by use of Takara SYBR Green Mastermix kit (Tianjin, China). TaqMan miRNA realtime PCR kit (ThermoFisher Scientific, USA) is applied to determine miR-6089 expression by the protocol. Primers for human genes are listed as follows: CircRNA_09505, (F): GTGTGACCTCCACAGCTACC, (R): TCACCAGAC ACACTGTGAGG; IL-6, (F): 5'-3' TGAACTCCTTCTC CACAAGC, (R): $5^{\prime}-3^{\prime}$ CTGAAGAGGTGAGTGGCTGT; TNF- $\alpha$ : (F): $5^{\prime}-3^{\prime}$ ATGTGGCAAGAGATGGGGAA, (R): 5'-3' CTCACACCCCACATCTGTCT; IL-12, (F): CTTG GTTTTCCCTGGTTTTT, (R): CTTTGACTTGGATGG TCAGG; AKT1, (F): GACGGGCACATTAAGATCAC, (R): TGAGGATGAGCTCAAAAAGC; GAPDH, (F): AA GGAAATGAATGGGCAGCC, (R): TAGGAAAAGCAT CACCCGGA.

\section{Cell proliferation and cell-cycle assay}

In brief, $2 \times 10^{5} /$ well THP- 1 macrophages are seeded into cell culture plates for 12, 24, and $48 \mathrm{~h}$. Cell counting kit-8 (CCK-8) kit (Vazyme Biotech, Nanjing, China) and 5-ethynyl-2'-deoxyuridine (EdU) kit (RiboBio, Guangzhou, China) are used to determine the proliferation of LV-circRNA, sh-circRNA, and control macrophages. After RNase treatment, the cell cycle is estimated by FACSCalibur (BD Biosciences, San Jose, CA, USA) incubating with propidium iodide (Keygen Biotech) at $37^{\circ} \mathrm{C}$ for $30 \mathrm{~min}$. All experiments are performed at least three times.

\section{Cytokine assay}

Human IL-6, TNF- $\alpha$, IL-12, IL-1 $\beta$, and IL- 8 in the supernatant of macrophages are measured by Enzymelinked Immunosorbent Assay (ELISA) according to the protocol of kits (R\&D Systems, USA). IL- 6 and TNF- $\alpha$ in mouse serum samples are determined by ELISA kits based on the manufacturer's instructions. Anti-mouse IL-4 and TNF- $\alpha$ monoclonal antibodis (eBioscience, San Diego, California, USA) were used to detect the intracellular cytokines of mouse synovial macrophages by flow cytometry.

\section{Western blot}

Cells are treated with cell lysis buffer containing 1 ul of protease inhibitor and 1ul of phenylmethanesulfonyl fluoride. After denaturation, protein samples are subjected to SDS PAGE for isolation and then are transferred to PVDF membranes by electroblotting. Primary antibodies of $\mathrm{AKT} 1, \mathrm{p}-\mathrm{AKT} 1, \mathrm{p}-\mathrm{I} \kappa \mathrm{B} \alpha, \mathrm{p}-\mathrm{NF}-\mathrm{kB}$, 훽actin, and GAPDH (Cell Signaling Technology, MA, USA) are used to incubate with PVDF membranes, which are subsequently incubated with HRP-conjugated secondary antibodies (Proteintech, USA). The enhanced chemiluminescence kit (Cell Signaling Technology, MA, USA) is applied for detection.

\section{Hematoxylin-eosin (H-E), toluidine blue, and safranin $\mathrm{O}$ staining and immunohistochemistry (IHC)}

Mice joint tissues are decalcificated by use of $10 \%$ EDTA, which is then embedded in paraffin for slides. $\mathrm{H}-\mathrm{E}$, Toluidine blue, and Safranin O (Solarbio) of knee joint sections are performed for detection of joint and cartilage damages. IHC was also carried out as previously described $^{31}$. The slides are incubated overnight with $\mathrm{p}$ AKT1 and p-NF- $\kappa B$ antibodies (Cell Signaling Technology, MA, USA).

\section{Fluorescence in situ hybridization (FISH) assay}

The location of circRNA_09505 in macrophages is determined by FISH. Macrophages are fixed with $4 \%$ paraformaldehyde and gradiently dehydrated with ethanol. Fluorescent-labeled probe for circRNA_09505 is applied during hybridization. We use DAPI (Beyotime, Shanghai, China) to stain the nucleus of macrophages.

\section{Luciferase reporter assay}

293T cells are used to estimate the targets of circRNA_09505 and miR-6089. Cells are transfected with miRNA mimics, inhibitors, AKT1-WT, AKT1-MUT, circRNA-WT, circRNA-MUT, or the corresponding plasmids with lipofectamine 2000. Macrophages are lysed and the luciferase activity is determined by Picagene Dual SeaPansy luminescence kit (Toyo Inc., Japan) based on the manufacturer's instructions as reported previously.

\section{RNA-binding protein immunoprecipitation}

RIP is preformed as described previously ${ }^{24}$. In brief, we perform experiments according to the protocol of Magna RIP Kit (Millipore, Bedford, USA). Cell lysates are immunoprecipitated using Ago2 antibody or IgG. The expression of circRNA_09505 and miR-6089 in immunoprecipitates is detected by real-time PCR as previously described, respectively.

\section{Statistical analysis}

The arthritis scores are analyzed by Mann-Whitney $U$ test between two groups. For other analyses, one-way ANOVA or Student's t test is applied for estimation. SPSS 16.0 (SPSS Inc., Chicago, USA) and Graphpad 5.0 software are used. $P<0.05$ is regarded to be statistically significant.

\footnotetext{
Acknowledgements

This study is supported by funds from the National Natural Science Foundation, China (81870237, 81801554, and 81601408), Development Plan of Excellent Youth Innovation Team in Shandong Province (2019), Shandong Natural Science Foundation (ZR2019QH012), and Weifang Science and
} 
Technology Development Program, Shandong Province, China (2019GX031, 2019YX020, and 2017YX019).

\section{Author details}

'Department of Rheumatology \& Central Laboratory of the First Affiliated Hospital, Weifang Medical University, Weifang 261053, China. ${ }^{2}$ Department of Physiology, Clinical Medicine College, Weifang Medical University, Weifang 261053, China. ${ }^{3}$ Department of Rheumatology and Immunology, Nanjing First Hospital, Nanjing Medical University, Nanjing 210006, China. ${ }^{4}$ Department of Gastrointestinal and Anal Diseases Surgery of the Affiliated Hospital, Weifang Medical University, Weifang 261053, China

\section{Author contributions}

All authors have participated in drafting the paper and approved the final version to be published. D.H. Xu and S.S. Yan took the responsibility for study design. Y.H. Yang, B.J. Gu, and J.H. Wang performed the experiments, data acquisition, and the data analysis. M. Cheng took the responsibility for interpretation of the data.

\section{Data availability}

All data are available upon request of the corresponding author.

\section{Conflict of interest}

The authors declare that they have no conflict of interest.

\section{Publisher's note}

Springer Nature remains neutral with regard to jurisdictional claims in published maps and institutional affiliations.

Supplementary Information accompanies this paper at (https://doi.org/ 10.1038/s41419-020-03038-z).

Received: 24 May 2020 Revised: 17 September 2020 Accepted: 21 September 2020

Published online: 07 October 2020

\section{References}

1. Catrina, A. I., Joshua, V., Klareskog, L. \& Malmstrom, V. Mechanisms involved in triggering rheumatoid arthritis. Immunol. Rev. 269, 162-174 (2016).

2. Klareskog, L., Lundberg, K. \& Malmstrom, V. Autoimmunity in rheumatoid arthritis: citrulline immunity and beyond. Adv. Immunol. 118, 129-158 (2013)

3. Udalova, I. A., Mantovani, A. \& Feldmann, M. Macrophage heterogeneity in the context of rheumatoid arthritis. Nat. Rev. Rheumatol. 12, 472-485 (2016).

4. Ma, W. T., Gao, F., Gu, K. \& Chen, D. K. The role of monocytes and macrophages in autoimmune diseases: a comprehensive review. Front. Immunol. 10, 1140 (2019).

5. Murray, P. J. Macrophage polarization. Annu. Rev. Physiol. 79, 541-566 (2017).

6. Piccolo, V. et al. Opposing macrophage polarization programs show extensive epigenomic and transcriptional cross-talk. Nat. Immunol. 18, 530-540 (2017).

7. Chen, W., Liu, D., Li, Q. Z. \& Zhu, H. The function of ncRNAs in rheumatic diseases. Epigenomics 11, 821-833 (2019).

8. Chen, J. Q., Papp, G., Szodoray, P. \& Zeher, M. The role of microRNAs in the pathogenesis of autoimmune diseases. Autoimmun. Rev. 15, 1171-1180 (2016).

9. Roy, S. \& Awasthi, A. Emerging roles of noncoding RNAs in T cell differentiation and functions in autoimmune diseases. Int. Rev. Immunol. 38 232-245 (2019).
10. Cosenza, S. et al. Pathogenic or therapeutic extracellular vesicles in rheumatic diseases: role of mesenchymal stem cell-derived vesicles. Int. J. Mol. Sci. 18, 889 (2017).

11. Tofino-Vian, M., Guillen, M. I. \& Alcaraz, M. J. Extracellular vesicles: a new therapeutic strategy for joint conditions. Biochem. Pharmacol. 153, 134-146 (2018).

12. Zhou, Z., Sun, B., Huang, S. \& Zhao, L. Roles of circular RNAs in immune regulation and autoimmune diseases. Cell Death Dis. 10 503 (2019).

13. Zheng, F., Yu, X., Huang, J. \& Dai, Y. Circular RNA expression profiles of peripheral blood mononuclear cells in rheumatoid arthritis patients, based on microarray chip technology. Mol. Med. Rep. 16, 8029-8036 (2017).

14. Li, B. et al. Hsa_circ_0001859 regulates ATF2 expression by functioning as an MiR-204/211 sponge in human rheumatoid arthritis. J. Immunol. Res. 2018, 9412387 (2018)

15. Xu, D. et al. Exosome-encapsulated miR-6089 regulates inflammatory response via targeting TLR4. J. Cell Physiol. 234, 1502-1511 (2019).

16. Mousavi, M. J. et al. Implications of the noncoding RNAs in rheumatoid arthritis pathogenesis. J. Cell Physiol. 234, 335-347 (2018).

17. Luo, Q. et al. Identification of circular RNAs hsa_circ_0044235 in peripheral blood as novel biomarkers for rheumatoid arthritis. Clin. Exp. Immunol. 194 118-124 (2018).

18. Yang, $X$. et al. Aberrant dysregulated circular RNAs in the peripheral blood mononuclear cells of patients with rheumatoid arthritis revealed by RNA sequencing: novel diagnostic markers for RA. Scand. J. Clin. Lab. Investig. https://doi.org/10.1080/00365513.2019.1674004 (2019).

19. Salmena, L. et al. A ceRNA hypothesis: the Rosetta Stone of a hidden RNA language? Cell 146, 353-358 (2011).

20. Li, L. J. et al. Competitive endogenous RNA network: potential implication for systemic lupus erythematosus. Expert Opin. Ther. Targets 21, 639-648 (2017).

21. Karreth, F. A. \& Pandolfi, P. ceRNA cross-talk in cancer: when ce-bling rivalries go awry. Cancer Discov. 3, 1113-1121 (2013).

22. Jiang, $\mathrm{H}$. et al. Reconstruction and analysis of the IncRNA-miRNA-mRNA network based on competitive endogenous RNA reveal functional IncRNAs in rheumatoid arthritis. Mol. Biosyst. 13, 1182-1192 (2017)

23. Xie, Z. et al. PU.1 attenuates TNFalphainduced proliferation and cytokine release of rheumatoid arthritis fibroblastlike synoviocytes by regulating miR155 activity. Mol. Med. Rep. 17, 8349-8356 (2018).

24. Yan, S. et al. Long non-coding RNA HIX003209 promotes inflammation by sponging miR-6089 via TLR4/NF-kappaB signaling pathway in rheumatoid arthritis. Front. Immunol. 10, 2218 (2019).

25. Su, Q. \& Lv, X. Revealing new landscape of cardiovascular disease through circular RNA-miRNA-mRNA axis. Genomics. https://doi.org/10.1016/j. ygeno.2019.10.006 (2019).

26. Cortes, R. \& Forner, M. J. Circular RNAS: novel biomarkers of disease activity in systemic lupus erythematosus? Clin. Sci. 133, 1049-1052 (2019).

27. Jia, N. et al. CeRNA expression profiling identifies KIT-related circRNA-miRNAmRNA networks in gastrointestinal stromal tumour. Front. Genet. 10, 825 (2019).

28. Zhang, Y., Li, X., Zhang, M. \& LV, K. Microarray analysis of circular RNA expression patterns in polarized macrophages. Int. J. Mol. Med. 39, 373-379 (2017).

29. Yang, X. et al. Silica-induced initiation of circular ZC3H4 RNAVZC3H4 pathway promotes the pulmonary macrophage activation. FASEB J. 32, 3264-3277 (2018).

30. Deng, T. et al. Calcitonin generelated peptide induces IL6 expression in RAW264.7 macrophages mediated by mmu_circRNA_007893. Mol. Med. Rep. 16, 9367-9374 (2017)

31. Wang, $X$. et al. Aggravated liver injury but attenuated inflammation in PTPROdeficient mice following LPS/D-GalN induced fulminant hepatitis. Cell Physiol. Biochem. 37, 214-224 (2015). 